\title{
Modelo de mejora de la competitividad basada en indicadores críticos de gestión en las pequeñas empresas de servicios de mantenimiento de equipos pesados
}

\section{RESUMEN}

El presente artículo busca incrementar la competitividad de las pequeñas empresas que brindan el servicio de mantenimiento de maquinaria pesada, mediante el diseño de un modelo basado en indicadores de gestión que derivan de los procesos críticos de este servicio; es decir, aquellos procesos de la cadena de valor que generan mayor competitividad para la empresa.

Con ello, se espera contribuir a elevar la competitividad de las empresas de este rubro y fomentar la investigación para elaborar herramientas o modelos que ayuden a sofisticar, poco a poco, la gestión de las mypes ( $98 \%$ de las empresas peruanas), mejorando la competitividad del sector empresarial del país.

Palabras clave: indicadores de gestión, procesos críticos, competitividad

MODEL BASED ON IMPROVED COMPETITIVENESS INDICATOR CRITICAL TO MANAGEMENT IN SMALL COMPANIES SERVICE OFFTERS MOINTENANCE OF CACHETERS HEAVY

\section{ABSTRACT}

This investigation seeks to increase the competitiveness of small companies that provide maintenance services for heavy equipment by designing game del based on indicators derived from the critical processes of this service, that is, those chain processes value, leading to greater competitive ness for the company.

With what is expected to help raise the competitiveness of companies in this sector and encourage research to develop tools or more sophisticated models that will help little by little, the management of MSE $(98 \%$ of Peruvian companies), improving the competitiveness country's business sector.

Keywords: management indicator, critical processes, competitiveness

\section{INTRODUCCIÓN}

El Perú tiene debilidades muy arraigadas, si no se mejoran en poco tiempo harían retroceder al país respecto a las mejoras alcanzadas; dentro de las principales debilidades que se identificaron, Porter (2010) mencionó que "la dificultad que tienen las empresas para alcanzar el desarrollo es debido a la baja competitividad entre ellas". Sobre este punto, adicionalmente expresó: "El Perú, no tiene una política ni una estrategia de largo plazo en temas de competitividad y, por tanto, es una economía que no tiene un rumbo definido. Esa responsabilidad no solo es del Gobierno, sino también de la población en general y de los empresarios en especial."

En el documento escrito por FENAPI (2005) sobre "El Análisis de la Competitividad de las Empresas" establece como parte de sus conclusiones "la existencia de problemas en la gestión administrativa de los procesos de las MYPE".

\subsection{Objetivo general}

Proponer un modelo de mejora de competitividad basada en indicadores críticos de gestión en las pequeñas empresas de mantenimiento de equipos pesados.

\subsection{Objetivos específicos}

a. Identificar y valorar la aplicación de instrumentos de gestión como herramientas de desarrollo y competitividad de las pequeñas empresas del sector servicio en el rubro de mantenimiento de equipos pesados.

b. Identificar los diferentes indicadores de gestión que viene aplicando el sector servicios de mantenimiento a maquinarias pesadas en sus pequeñas empresas y su repercusión en la competitividad.

c. Formular indicadores de gestión básicos y sencillos que permitan incidir en la línea del proceso, en busca de elevar la competitividad en este sector de empresas.

d. Expresar con fundamento estadístico, la competitividad de las pequeñas empresas del sector en función a los indicadores de gestión formulados.

* Docente Principal, investigadora del Departamento Académico de Producción y Gestión Industrial de la UNMSM, Facultad de Ing. Industrial, del curso de Gestión de Mype. Asesora de la tesis. E-mail: teogaza57@yahoo.es

** Docente Asociado de la UNMSM, de la Facultad de Ciencias Administrativas. E-mail: csotomayorsd@hotmail.com 


\section{MARCO TEÓRICO}

\subsection{Antecendentes del problema}

El tema de investigación está vinculado al sector servicio, particularmente al mantenimiento de equipos pesados.

En los antecedentes de las mype de servicios se distingue un primer Marco Global (SWISS CONTACT, 2005) donde se encuentra información elaborada por organizaciones y especialistas de otros países que en su realidad nacional o regional el sector servicios y las mype también tienen gran protagonismo a nivel económico, esto con el objetivo de entender la evolución y contribución de las mype en el desarrollo de otras economías; sustentadas en estudios de la fundación Suiza, (ALERTA ECONÓMICA, MAXIMIXE, 2009), y un segundo marco nacional, que se compone de información específicamente de las mype del país y su evolución en los últimos años.

El mercado de mantenimiento de equipos pesados que prestan servicios al sector construcción, minería, saneamiento y otros en Perú, ha venido creciendo en la misma medida que la incorporación de nuevos equipos por las empresas líderes al mercado nacional, ante el impulso y desarrollo de estos sectores en el Perú; en tal razón, las mype de este sector han emergido como consecuencia de las necesidades del mercado, incorporándose nuevas unidades económicas, anteponiendo su destreza técnica y soslayando la gestión de los procesos, lo que le ha restado competitividad.

\subsection{Ventajas del sistema}

El estudio busca elevar la competitividad y la productividad para este sector de empresas, las mismas que son intensivas en mano de obra calificada, lo que tendrá incidencia en el aspecto social del sector y por ende del país.

Esta mejora de la competitividad comprende la revisión de los procesos y el planteamiento de utilización de indicadores, lo que repercutirá en la mejora de la organización y se espera un aporte tangible para el sector.

Con esta investigación se podrá conocer los procesos críticos de la empresas y los indicadores para su monitoreo y control, basada en el diseño de un modelo, asentando un valor teórico en la ciencia del conocimiento, que puede servir como base para empresas de sectores diferentes que requieran revisar sus procedimientos de gestión.

\subsection{Aplicaciones}

En las pequeñas y microempresas, para no permanecer rezagadas por empresas de mayor tamaño deberán invertir recursos en aplicar herramientas de gestión, esto generará competitividad independientemente de la actividad económica a la que se dediquen.

El modelo presentado, basado en indicadores de gestión no solo debe ser empleado para medir el nivel de competitividad de las empresas, es decir la etapa de diagnóstico del problema; sino también debe ser aplicado como guía para la elaboración de planes y programas que busquen mejorar la rentabilidad. Cabe mencionar que la identificación de un problema no es más que el inicio de un proceso de cambios en busca del progreso.

\section{FORMULACIÓN DEL PROBLEMA}

¿En qué medida la utilización de indicadores de gestión en los procesos críticos de las pequeñas empresas del sector servicio de mantenimiento de equipos pesados les permiten mejorar su competitividad?

\section{PLANTEAMIENTO DE HIPÓTESIS}

\subsection{Hipótesis y variables de la investigación}

Las hipótesis planteadas constan de una general y cinco hipótesis específicas, que serán verificadas mediante las técnicas estadísticas, por ello será preciso plantear hipótesis nulas $\left(\mathrm{H}_{0}\right)$ e hipótesis alternativas $\left(\mathrm{H}_{1}\right)$ :

\subsection{Hipótesis general}

La aplicación de indicadores de gestión en base a procesos críticos, en las pequeñas empresas dedicadas al servicio de mantenimiento de equipos pesados incrementará sus niveles de competitividad, lo que incide directamente en la elevación económica de estas empresas, así como del sector servicios y por ende del país; se plantea:

$\mathbf{H}_{\mathbf{0}}$ : "La aplicación de indicadores de gestión en los procesos críticos de mantenimiento, en las pequeñas empresas de servicio pesado no incrementa el nivel de competitividad."

$\mathbf{H}_{1}$ : "La aplicación de indicadores de gestión en los procesos críticos de mantenimiento en las pequeñas empresas de servicio incide favorablemente en la mejora de su competitividad." 


\section{Identificación de variables}

\section{Variables independientes:}

V.I.: Gestión de procesos críticos

Indicadores:

- Integridad del diagnóstico

- Efectividad del control de la calidad

- Seguimiento del servicio (postservicio)

\section{Variables dependientes:}

V.D.: Nivel de competitividad de la empresa

\section{Indicador:}

- Nivel de ingresos mensuales

\section{MÉTODOS Y RESULTADOS}

El estudio de investigación se orienta hacia el tipo de investigación "no experimental", no se manipulan las variables intencionalmente, sino se observan los fenómenos tal y como se dan en su contexto natural, se ha seleccionado el método "correlacional", debido a que la investigación busca relacionar la calidad de la gestión de los procesos críticos de las empresas del sector en estudio, con su incidencia sobre la competitividad de estas empresas.

Unidad de análisis: los procesos críticos de cada una de las pequeñas empresas dedicadas al servicio de mantenimiento de maquinaria pesada que operan en la región Lima Metropolitana y alrededores, al año 2010.

Población de mantenimiento de maquinaria pesada que operaban en la región Lima Metropolitana y Callao hasta el año 2010, para definir la población, se trabajó con dos métodos diferentes y luego mediante aproximaciones sucesivas entre los dos resultados se determina el tamaño de la población de la investigación.

$1^{\circ}$ Método: Estimación del tamaño de la población con datos estadísticos se desarrolla en la Tabla 1.

De lo que se desprende que las pequeñas empresas dedicadas al mantenimiento de maquinaria en Lima, es decir la población a investigar, es aproximadamente de 342 empresas.

Tabla 1. Determinación de la población según el $1^{\circ}$ Método

\begin{tabular}{|c|c|c|}
\hline Variable & $\begin{array}{l}\text { Número de } \\
\text { empresas }\end{array}$ & Determinación del dato \\
\hline $\mathrm{T}=$ Todas las empresas en el Perú & 943275 & (INEI, 2011, p. 23), "Características económicas de las MYPE" \\
\hline $\begin{array}{l}\mathrm{R}=\text { Todas las empresas dedicadas a } \\
\text { reparación e instalación de máquinas y } \\
\text { equipos en el Perú }\end{array}$ & 1800 & $\begin{array}{l}\text { (INEI, 2009, p. 26), "Principales características de los establecimiento } \\
\text { de las industrias manufactureras", basado en el último censo } \\
\text { económico nacional. }\end{array}$ \\
\hline $\begin{array}{l}\mathrm{L}=\text { Todas las empresas de reparación } \\
\text { e instalación de máquinas y equipo } \\
\text { ubicadas en Lima y Callao (estimación) }\end{array}$ & 760 & $\begin{array}{l}\text { ( R ) } \times(\% \text { Lima + Callao ) = P } \\
(1,800) \times(3,4 \%+2,8 \%)=720 \\
\% \text { Lima + Callao = porcentaje de todas las empresas del Perú, que } \\
\text { se ubican en Lima (INEI, 2008, p. 20). "Resultados del IV censo } \\
\text { económico nacional". El último censo hasta la actualidad. }\end{array}$ \\
\hline $\begin{array}{l}P=\text { Pequeñas empresas dedicadas a } \\
\text { reparación e instalación de maquinarias } \\
\text { y equipos }\end{array}$ & 342 & $\begin{array}{l}\text { (P) } \times(\% \text { P.E. })=\text { P } \\
(760) \times(45 \%)=342 \\
\% \text { P.E. = porcentaje de las empresas que son pequeña empresa. } \\
\text { - } \quad \text { (INEI, 2011, 23) "Características económicas de las MYPE", el } \\
\text { porcentaje de empresas pequeñas a nivel nacional es del } 4 \% \text { y } \\
\text { micro } 95,1 \% \text {. } \\
\text { - Sin embargo, empresarios dedicados a la actividad conocedores } \\
\text { de las características de la competencia creen que él \% de la } \\
\text { pequeña empresa en esta actividad es mucho mayor, pues las } \\
\text { características del negocio hacen necesarias mayor nivel de } \\
\text { inversión y más personal. De lo que se estima que el porcentaje } \\
\text { de pequeñas empresas es de } 45 \%\end{array}$ \\
\hline
\end{tabular}

Fuente: INEI, Elaboración propia. 
$2^{\circ}$ Método: Estimación del tamaño de la población basado los registros municipales de empresas por distrito. Se seleccionan los nueve distritos con zonas industriales más importantes de Lima Metropolitana, contabilizándose las empresas dedicadas al mantenimiento de maquinaria y equipos. La Tabla 2 muestra resumidamente el proceso y los resultados.
Estas municipalidades registran un total de aproximadamente 300 empresas que pueden calificar con el perfil de la población.

Entonces, por aproximaciones sucesivas entre los dos métodos se llega a determinar que la población es aproximadamente de 320 pequeñas empresas, tal como lo muestra la Tabla 3.

Tabla 2. Población de la Investigación según el $2^{\circ}$ Método

\begin{tabular}{|l|l|c|}
\hline \multirow{2}{*}{ Distritos incluidos } & \multicolumn{2}{|c|}{ 495 Empresas } \\
& San Juan de Lurigancho y La Victoria \\
& Alquiler de maquinaria & 4 \\
\cline { 2 - 3 } & Alquiler de maquinaria pesada & 7 \\
\cline { 2 - 3 } & Fabricación de carrocerías para vehículos & 2 \\
\cline { 2 - 3 } Rubros incluidos & Fabricación de maquinaria & 1 \\
\cline { 2 - 3 } & Mantenimiento y reparación de equipos y máquinas mayores & 237 \\
\cline { 2 - 3 } & Mantenimiento de maquinaria & 44 \\
\cline { 2 - 3 } & Mantenimiento de maquinaria pesada & 17 \\
\cline { 2 - 3 } & Servicio de mecánica menor & 24 \\
\cline { 2 - 3 } & Venta de maquinaria pesada & 34 \\
\cline { 2 - 3 } & Venta de maquinaria & 13 \\
\cline { 2 - 3 } & Venta de partes, piezas y repuestos & 109 \\
\hline & Venta de vehículos automotores & 2 \\
\hline & Venta de vehículos automotores & 2 \\
\cline { 2 - 3 } & Venta y mantenimiento de vehículos & 2 \\
\hline
\end{tabular}

Fuente: Registros municipales. Elaboración propia.

Tabla 3. Población de la investigación

\begin{tabular}{|c|c|c|}
\hline $2^{\circ}$ Método & Aproximaciones sucesivas & $1^{\circ}$ Método \\
\hline 300 empresas & 320 empresas & 342 empresas \\
\hline
\end{tabular}

Fuente: Elaboración propia. 
Tamaño de la población: las 320 pequeñas empresas dedicadas al mantenimiento de maquinaria y equipo pesado en Lima Metropolitana.

Tamaño de la muestra: en base al método propuesto por Newbold, Carlson y Thorne (2008) se llega a determinar que el tamaño de la muestra, partiendo de una muestra preliminar; alcanza 54 pequeñas empresas de mantenimiento de maquinaria pesada.

Selección de la muestra: luego de obtenido el tamaño de la muestra, mediante números aleatorios se seleccionaron las 54 empresas que integran la muestra del total de empresas que forman parte de la lista confeccionada con la información de las municipalidades.

\subsection{Resultados}

Con la finalidad de responder a la pregunta y los objetivos de la investigación, se llevaron a cabo cursos de acción que permitieron demostrar las hipótesis y formular el modelo basado en indicadores de gestión.

La investigación busca demostrar que para mejorar la competitividad, las empresas de este sector deberán gestionar de forma eficiente ciertos procesos en especial, aquellos que generan mayor valor agregado al servicio.

Luego de planteados los procesos críticos e indicadores, mediante un minucioso análisis cualitativo, se establece las mediciones de cada uno de estos. Seguidamente se hace el análisis estadístico que permite corroborar las hipótesis, dándoles fundamento a los indicadores planteados, además de diseñar el modelo que mejor se ajusta a los datos obtenidos de la encuesta y expresar cuantitativamente la relación entre las dos variables, nivel de competitividad y gestión de procesos críticos.

\section{Análisis e interpretación de la información (Análisis cualitativo)}

\section{Planteamiento de indicadores}

Para seleccionar los procesos críticos es necesario entender la cadena de valor de estas empresas e identificar los procesos que se pueden reconocer como críticos, para luego traducirlos en indicadores de gestión; de la siguiente forma:

Cadena de valor genérica de las pequeñas empresas de servicios de mantenimiento de equipos pesados.

Con el propósito de profundizar la cadena de valor se desarrolla la metodología del análisis del valor que se basa en los costos de los procesos, y como en este caso el costo varía según el tipo de mantenimiento, máquina o falla, se tomará la variable horas/hombre invertidas en cada proceso. El método responde a cuestionamientos, de cuyas respuestas se desprende el análisis de valor presentada en la Tabla 4.

De la Tabla 4, se pueden identificar que los procesos más importantes para lograr que el servicio funcione y satisfaga al cliente se resume en la Tabla 5.

Empezando por los procesos operativos, el diagnóstico debe ser mejorado por las pequeñas empresas imprimiéndole mayor valor, que a largo plazo resulta siendo un ahorro para el usuario y una mejora de la competencia para la empresa.

En cuanto al control de la calidad, proceso raramente aplicado por las pequeñas empresas, consiste en el seguimiento mientras se realiza el mantenimiento, y la realización de pruebas una vez culminado el mantenimiento, para asegurar que se hayan superado las fallas y para identificar cualquier avería futura que esté presentando síntomas.

Postservicio, también considerado como primario. Ciertas empresas grandes de este sector se han logrado posicionar tomando como estrategia de diferenciación basada en el postservicio; es decir mantener comunicación con el cliente.

Puesto que, el proceso de mantenimiento es difícil de diferenciarse de una empresa a otra, ya que la calidad técnica de las empresas pequeñas del sector es parejamente aceptable.

En base a estas consideraciones se propusieron diversas ideas para mejorar los procesos; para luego seleccionar solo las que cumplen con el requisito de viabilidad; tales como:

\section{Evaluación y selección:}

Con ayuda de la información obtenida de las visitas a TECSUP, centro de estudios especialista en mantenimiento de maquinaria pesada; se evaluaron las propuestas creativas y se establecieron las actividades que deben comprender el diagnóstico, el mantenimiento en sí y el control de calidad para las pequeñas empresas: diagnóstico, mantenimiento, control de calidad.

\section{A) Selección de los procesos críticos para la competitividad}

Habiendo entendido y definido todos los procesos necesarios para el eficiente desempeño de las pequeñas empresas en el servicio de mantenimiento de maquinaria pesada, se secuenciaron los procesos; como se puede apreciar en el Gráfico 1. 
Tabla 4. Análisis de Valor

\begin{tabular}{|c|c|c|}
\hline \multirow[b]{2}{*}{ PRODUCTO } & \multicolumn{2}{|c|}{ Un servicio de mantenimiento de maquinaria pesada } \\
\hline & \multicolumn{2}{|c|}{$\begin{array}{l}\text { Necesidad que satisface: mantener las maquinarias de otras empresas en buen y } \\
\text { constante funcionamiento }\end{array}$} \\
\hline \multirow{4}{*}{ FUNCIONES DEL SERVICIO } & \multicolumn{2}{|l|}{ F1: Mantenimiento prevención (principal) } \\
\hline & \multicolumn{2}{|l|}{ F2: Mantenimiento corrección (secundaria) } \\
\hline & \multicolumn{2}{|l|}{ F3: Mantenimiento paliativo (secundaria) } \\
\hline & \multicolumn{2}{|l|}{ F4: Mantenimiento predictivo (secundaria) } \\
\hline \multirow{7}{*}{$\begin{array}{l}\text { FUNCIÓN PRINCIPAL: } \\
\text { MANTENIMIENTO PREVENTIVO }\end{array}$} & PROCEDIMIENTOS & HORAS/HOMBRE \\
\hline & 1. Diagnóstico & 1 hora/hombre \\
\hline & 2. Presupuesto & $1 / 2$ hora/hombre \\
\hline & 3. Compra de insumos & $1 / 2$ hora/hombre \\
\hline & 4. Mantenimiento & 7 horas/hombre \\
\hline & 5. Control de calidad & $1 / 2$ hora/hombre \\
\hline & 6. Seguimiento del servicio (postservicio) & 1 hora/hombre \\
\hline \multirow{6}{*}{$\begin{array}{l}\text { FUNCIÓN SECUNDARIA 1: } \\
\text { MANTENIMIENTO CORRECTIVO }\end{array}$} & PROCEDIMIENTOS & HORAS/HOMBRE \\
\hline & 1. Diagnóstico & 3 horas/hombre \\
\hline & 2. Presupuesto & 1 hora/hombre \\
\hline & 3. Compra de materiales requeridos & 1 hora/hombre \\
\hline & 4. Mantenimiento & 6 horas/hombre \\
\hline & 5. Control de calidad & $1 \frac{1}{2}$ horas/hombre \\
\hline \multirow{6}{*}{$\begin{array}{l}\text { FUNCIÓN SECUNDARIA 2: } \\
\text { MANTENIMIENTO PREDICTIVO }\end{array}$} & PROCEDIMIENTOS & HORAS/HOMBRE \\
\hline & 1. Seguimiento del servicio (postservicio) & 2 horas/hombre \\
\hline & 2. Presupuesto & $1 / 2$ hora/hombre \\
\hline & 3. Compra de materiales requeridos & $1 / 2$ hora/hombre \\
\hline & 4. Mantenimiento & 2 horas/hombre \\
\hline & 5. Control de calidad & $1 / 2$ hora/hombre \\
\hline \multirow{7}{*}{$\begin{array}{l}\text { FUNCIÓN SECUNDARIA 3: } \\
\text { MANTENIMIENTO PALIATIVO }\end{array}$} & PROCEDIMIENTOS & HORAS/HOMBRE \\
\hline & 1. Comunicación con el cliente (postservicio) & 2 horas/hombre \\
\hline & 2. Diagnostico & 2 horas/hombre \\
\hline & 3. Presupuesto & 1 hora/hombre \\
\hline & 4. Compra de materiales requeridos & 1 hora/hombre \\
\hline & 5. Mantenimiento & 6 horas/hombre \\
\hline & 6. Control de calidad & $1 \frac{1}{2}$ hora/hombre \\
\hline
\end{tabular}

Fuente: Elaboración propia.

Tabla 5. Procesos en orden de prioridad

\begin{tabular}{|c|c|}
\hline PROCESO & $\mathbf{N} .^{\circ}$ HORAS/HOMBRE \\
\hline Mantenimiento & 21 horas/hombre \\
\hline Diagnóstico & 6 horas/hombre \\
\hline Control de calidad & 4 horas/hombre \\
\hline Seguimiento del servicio & 4 horas/hombre \\
\hline Presupuesto & 3 horas/hombre \\
\hline Compra de requerimientos & 3 horas/hombre \\
\hline Comunicación con el cliente & 2 horas/hombre \\
\hline
\end{tabular}

Fuente: Elaboración propia. 
Tabla 6. Cadena de valor completa

\begin{tabular}{|c|c|c|c|c|c|c|}
\hline \multicolumn{2}{|c|}{$\begin{array}{l}\text { ADMINISTRACIÓN Y FINANZAS } \\
\text { - Soporte administrativo } \\
\text { - Control de costos }\end{array}$} & $\begin{array}{l}\text { - Elaboración de fac } \\
\text { - Operaciones finan }\end{array}$ & $\begin{array}{l}\text { cturas y guías } \\
\text { cieras }\end{array}$ & & & \\
\hline \multicolumn{2}{|c|}{$\begin{array}{l}\text { - Contratación de personal } \\
\text { - Capacitación de personal }\end{array}$} & \multicolumn{3}{|c|}{$\begin{array}{l}\text { - Remuneración de personal } \\
\text { - Promoción de personal }\end{array}$} & \multicolumn{2}{|c|}{ - Relaciones interpersonales } \\
\hline \multicolumn{7}{|c|}{ ABASTECIMIENTO Y SERVICIOS GENERALES } \\
\hline COMPRAS & \multicolumn{4}{|c|}{ OPERACIONES } & $\begin{array}{l}\text { MARKETING Y } \\
\text { VENTAS }\end{array}$ & POSTSERVICIO \\
\hline $\begin{array}{l}\text {-Evaluación de } \\
\text { requerimientos } \\
\text {-Evaluación de } \\
\text { proveedores } \\
\text { - Elaboración } \\
\text { de ordenes de } \\
\text { compras }\end{array}$ & \begin{tabular}{l}
\multicolumn{1}{c|}{ DIAGNÓSTICO } \\
-Revisión de avería \\
y/o requerimiento \\
del Servicio \\
- Informe técnico del \\
diagnostico
\end{tabular} & \begin{tabular}{l}
\multicolumn{1}{|c|}{ PRESUPUESTO } \\
- Elaboración de \\
propuesta económica \\
- Entrega de \\
presupuesto de \\
servicio
\end{tabular} & \begin{tabular}{|l|} 
MANTENIMIENTO \\
-Mantenimiento \\
Preventivo \\
- Mantenimiento \\
Correctivo \\
- Mantenimiento \\
Predictivo
\end{tabular} & \begin{tabular}{|l}
\multicolumn{1}{|c}{ CONTROL DE } \\
CALIDAD \\
- Supervisión del \\
servicio \\
- Prueba del \\
Servicio
\end{tabular} & $\begin{array}{l}\text {-Promoción del } \\
\text { servicio } \\
\text {-Publicidad del } \\
\text { servicio } \\
\text {-Captación del } \\
\text { servicio } \\
\text {-Atención al cliente }\end{array}$ & $\begin{array}{l}\text {-Seguimiento del } \\
\text { servicio } \\
\text { - Comunicación al } \\
\text { cliente } \\
\text { - Recepción de } \\
\text { quejas y sugerencias }\end{array}$ \\
\hline
\end{tabular}

Fuente: Elaboración propia.

Gráfico 1. Flujograma del proceso

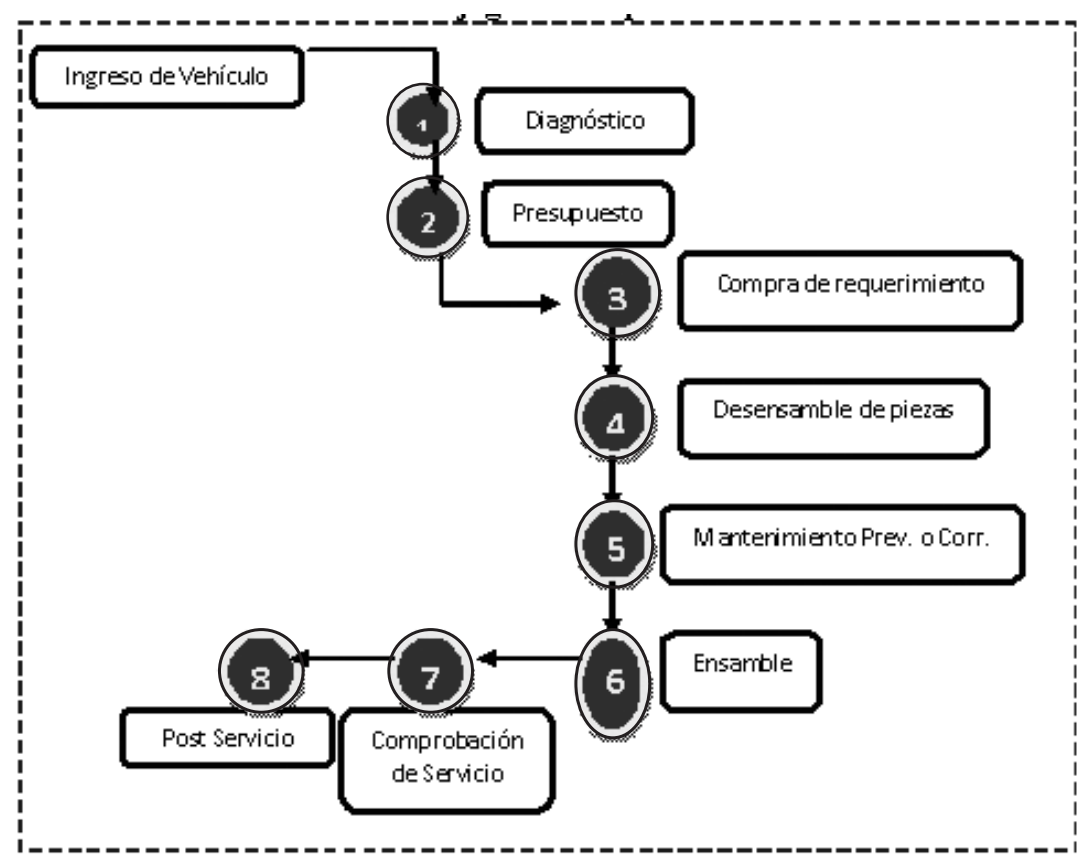

Fuente: Elaboración propia. 
Para identificar que procesos generan valor agregado es necesario evaluar cada uno de los procesos que se relacionan directamente con el resultado del servicio, mediante indicadores o criterios de priorización que reflejen que tanta competitividad genera cada uno; basado y considerando que Porter (2002) propuso que la competitividad industrial está basada en cinco fuerzas, se ha diseñado una matriz, presentada en la Tabla 7 para su evaluación.

Tabla 7. Ponderación de indicadores

\begin{tabular}{|l|c|}
\hline \multicolumn{1}{|c|}{ INDICADOR } & PESO \\
\hline Rivalidad con competidores & $30 \%$ \\
\hline Poder de negociación con proveedores & $20 \%$ \\
\hline Poder de negociación con compradores & $30 \%$ \\
\hline Amenaza de productos sustitutos & $10 \%$ \\
\hline Amenaza de nuevas entradas & $10 \%$ \\
\hline
\end{tabular}

Fuente: Elaboración propia.
Adicionalmente, se califica cada proceso en la medida que pueden permitir ventajas en cuanto a cada agente del mercado, ya sea por costo, diferenciación o segmentación; la puntuación se muestra en la Tabla 8.

Tabla 8. Calificación de competitividad de procesos

\begin{tabular}{|l|c|}
\hline \multicolumn{1}{|c|}{ Competitividad basada en: } & Puntaje \\
\hline Costos (C) & 1 \\
\hline Diferenciación (D) & 1 \\
\hline Segmentación (S) & 1 \\
\hline
\end{tabular}

Fuente: Elaboración propia.

Como se aprecia en la matriz, se ha seleccionado al diagnóstico como el proceso que constituye mayor fuente de competitividad, seguido del contro de calidad y el postservicio, logrando identificar los procesos críticos de la cadena de valor.

Tabla 9. Matriz de competitividad de procesos

\begin{tabular}{|c|c|c|c|c|c|c|c|}
\hline \multicolumn{2}{|c|}{ Indicadores } & \multirow[t]{2}{*}{ Diagnóstico } & \multirow[t]{2}{*}{ Presupuesto } & \multirow[t]{2}{*}{ Compras } & \multirow[t]{2}{*}{$\begin{array}{l}\text { Manteni- } \\
\text { miento }\end{array}$} & \multirow[t]{2}{*}{$\begin{array}{l}\text { Control de } \\
\text { Calidad }\end{array}$} & \multirow[t]{2}{*}{ Postservicio } \\
\hline Indicador & Peso & & & & & & \\
\hline Competidores & 0.3 & $(C)+(S)=2$ & & & & $(D)=1$ & $(D)=1$ \\
\hline Proveedores & 0.2 & & $(C)=1$ & $(C)+(S)=2$ & & & \\
\hline Compradores & 0.3 & $(C)+(S)=2$ & & & $(S)=1$ & $(C)+(D)=2$ & $(C)+(D)=2$ \\
\hline $\begin{array}{l}\text { Productos } \\
\text { Sustitutos }\end{array}$ & 0.1 & & & & & & \\
\hline $\begin{array}{l}\text { Nuevas } \\
\text { Entradas }\end{array}$ & 0.1 & & & & & & \\
\hline TOTAL & 1 & 1.2 & 0.2 & 0.4 & 0.3 & 0.9 & 0.9 \\
\hline
\end{tabular}

Fuente: Elaboración propia.

Todo este análisis sustentado desde el análisis del valor y la definición de los procesos, hasta su diagramación en el flujograma, análisis de la competitividad basada en teorías de Porter y la observación basada en la experiencia; se puede consolidar como un procedimiento general que logra entender los procesos e identificar los que son críticos o generadores de competitividad.

Así es como se puede entender basado en el análisis de la caja negra, como se muestra en la Gráfica 2. 
Gráfica 2. Análisis de la caja negra

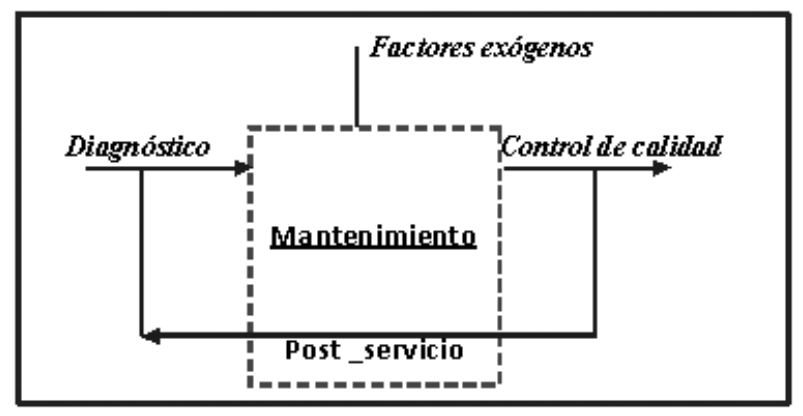

Fuente: Elaboración propia.

\section{Estructura del modelo de indicadores de gestión}

Este apartado se divide en dos títulos, el primero de ellos consiste en plantear las mediciones para los indicadores o procesos críticos seleccionados y el segundo, en plantear la estructura del modelo.

Medición de las variables independientes: este se desarrolló a través del diagnóstico (superficial, intermedio y profundo), el control de calidad (sin control de calidad, supervisor de calidad, prueba de calidad) y del postservicio (sin seguimiento, seguimiento esporádico y seguimiento permanente), se obtuvo el siguiente resultado: de todos los niveles de diagnóstico, control de calidad y postservicio, según su grado de profundidad y complejidad.

\section{PLANTEAMIENTO DE LA ESTRUCtURA DEL MODELO DE GESTIÓN}

Tal como ya se ha venido comentando, el presente estudio busca demostrar que la "Competitividad" (CPT) está en función a la variable "Gestión de procesos críticos". Esta última variable, de naturaleza independiente, que se postula puede medirse mediante tres indicadores que se basa en los procesos críticos de la cadena de valor; tal como se muestra en la Gráfica 3.

Para determinar los valores de los parámetros, que son constantes, se calculará cual es el peso ponderado de cada uno de los procesos críticos en la competitividad. Estos pesos se obtuvieron del análisis cuantitativo a la encuesta aplicada a las empresas de la muestra.

Para esto, se designó una calificación a cada nivel de complejidad con que se elaboran los procesos críticos.

Finalmente, la variable dependiente o competitividad se determina en base a un solo indicador; este es las ventas mensuales que alcanza la empresa. Para medir este indicador se toman dos subvariables que muestra la Gráfica 4.

Gráfica 3. Estructura del Modelo basado en indicadores de gestión

$\left\{\begin{array}{c}\text { CPT } \rightarrow f \text { (Procesos Crilicos) } \\ \Delta\end{array}\right.$
Gestión de Procesos Criticos $\rightarrow f(\alpha A, \beta B, \omega C)$
Donde:

Fuente: Elaboración propia

Gráfica 4. Cálculo del indicador de competitividad

$$
\begin{aligned}
& \text { CPT } \Rightarrow \text { Ingreso Mensual promedio } \\
& C P T \Rightarrow(\text { NPSM }) \text { (PPS) }
\end{aligned}
$$

\section{Donde:}

NPSM $=N^{n}$ promedio de servicios al mes

PPS = Precio promedio de servicios

Fuente: Elaboración propia.

Esta es la estructura del modelo de gestión para empresas de mantenimiento de maquinaria pesada, basándose en indicadores de gestión elaborados de sus procesos críticos.

\section{Prueba de hipótesis (análisis cuantitativo)}

Luego del análisis e interpretación de la información de carácter cualitativa que permitió proponer los indicadores de gestión, sus mediciones y diseñar la estructura del modelo de gestión, se complementa el análisis en el campo cuantitativo; para esto, se demuestran estadísticamente cada una de las hipótesis provenientes de los procesos críticos seleccionados que permitieron darle validez científica al modelo de gestión.

\section{Comprobación de representatividad de la muestra}

Se toman las características más importantes de identificación de la unidad de análisis, es decir el número de trabajadores con que cuenta cada empresa y su nivel de ventas anual. 
Hipótesis específica 3: Proceso de diagnóstico

$$
\begin{aligned}
\text { Siendo }: & \boldsymbol{r}=[-1 ; 1] \\
\mathrm{H}_{0}: & \left.r_{\text {Diag }} \approx 1 \text { (rseaproximaa } 1\right) \\
\mathrm{H}_{1}: & r_{\text {Diag }} \approx A(r \text { no scaproximaa } 1)
\end{aligned}
$$

De los datos obtenidos se extraen los puntajes de las 54 empresas en cuanto a la profundidad del diagnóstico que realizan, y los ingresos mensuales promedio que perciben; en base a ellos se realizó el análisis que indica un coeficiente de correlación casi nulo $(0,08)$; esto debido a que dentro de las empresas que cuentan con un diagnóstico casi inexistente se encuentran algunas que perciben bajos ingresos y otras con ingresos muy elevados.

Por tal motivo, es correcto promediar los ingresos de las empresas que pertenecen a un mismo nivel de diagnóstico, así se tiene la Gráfica 5 donde se entiende de mejor manera el comportamiento de los ingresos mensuales al incrementar la profundidad en el diagnóstico.

Gráfica 5. Relación entre nivel de diagnóstico e ingreso mensual en promedio

\begin{tabular}{|c|c|}
\hline Diagnóstico & Ingreso mensual promedio \\
\hline 1 & S/. 101,707 \\
\hline 2 & S/. 169,789 \\
\hline 6 & S/. 201,873 \\
\hline
\end{tabular}

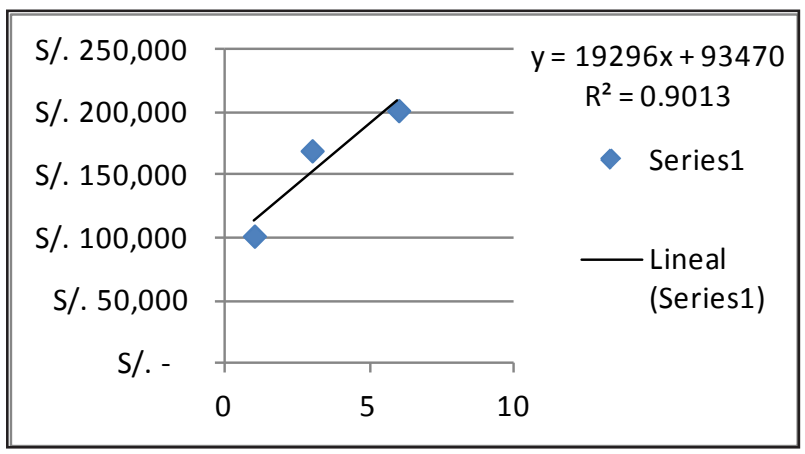

Fuente: Elaboración propia

En esta gráfica se aprecia un coeficiente de correlación de 0,9 positivo y bastante cercano a 1 ; es decir, la hipótesis ha sido aprobada.
Prosiguiendo con el proceso de control de calidad se ha planteado la siguiente hipótesis, muy similar a la anterior.

Hipótesis específica 4: Proceso Control de calidad

$$
\text { Siendo : } r=[-1 ; 1]
$$

$$
\begin{array}{ll}
\mathrm{H}_{0}: & \left.r_{\text {Cont.c. }} \approx 1 \text { (rseaproximaa } 1\right) \\
\mathrm{H}_{1}: & r_{\text {Cont.c. }} \approx 1(r \text { no seaproximaa } 1)
\end{array}
$$

Al igual que el proceso anterior, se hace el análisis estadístico promediando los ingresos de las empresas de la muestra. Así se tiene la Gráfica 6.

Gráfica 6. Relación entre el control de calidad e ingreso mensual en promedio

\begin{tabular}{|c|c|}
\hline Diagnóstico & Ingreso mensual promedio \\
\hline 1 & S/. 73,100 \\
\hline 3 & $S / .115,492$ \\
\hline 6 & $S / .337,447$ \\
\hline
\end{tabular}

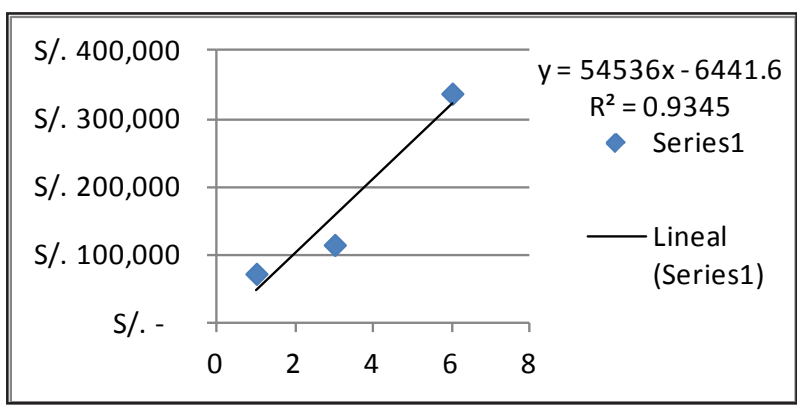

Fuente: Elaboración propia.

De la gráfica se entiende que el control de calidad según la profundidad con que se lleve a cabo genera incrementos en los ingresos de las empresas del sector en cuestión, ya que mantienen un coeficiente de correlación del 0,93, más cercano a 1 que el proceso anterior. En conclusión la hipótesis 4 ha sido corroborada.

Para finalizar con el segundo grupo de hipótesis específicas, se tiene la hipótesis 5 que hace referencia a la relación explicativa que se da entre el postservicio y el ingreso mensual promedio que tienen las empresas del sector. 
Hipótesis Específica 5: Proceso de postservicio

$$
\begin{aligned}
\text { Siendo }: r=[-1 ; 1] \\
\left.H_{0}: \quad r_{\text {Pst } S} \approx 1 \text { (rsecuproximaa } 1\right) \\
H_{1}: \quad r_{\text {Pst } S .} \approx 1(r \text { no secproximaa } 1)
\end{aligned}
$$

De los datos obtenidos se prepara la Gráfica 7 con el coeficiente de correlación, en donde se puede apreciar una relación fuerte de 0,84. Si bien es cierto el valor del coeficiente no es tan bueno como en la hipótesis anterior, sigue siendo un valor muy aceptable aprobándose.

Gráfica 7. Relación entre el postservicio y el ingreso mensual promedio

\begin{tabular}{|c|c|}
\hline Diagnóstico & Ingreso mensual promedio \\
\hline 1 & S/. 79,615 \\
\hline 3 & S/. 77,286 \\
\hline 6 & S/. 311,320 \\
\hline
\end{tabular}

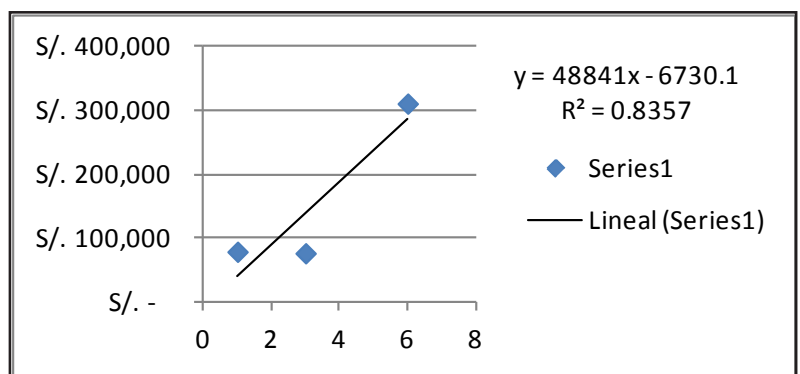

Fuente: Elaboración propia.

Luego de confirmar los procesos críticos se determinan los valores de los parámetros correspondientes a cada de los procesos, para ello se considera la frecuencia relativa porcentual ( $h i \%)$ que alcanzaron luego de tabular las respuestas de la pregunta 3 de la encuesta, tal como se aprecia en la Tabla 10.

Entonces, de acuerdo a los porcentajes alcanzados por cada proceso se establecen los valores de los parámetros para cada indicador explicado en la Tabla 11.
Tabla 10. Procesos por orden de prioridad, según expertos

\begin{tabular}{|l|c|}
\hline \multicolumn{1}{|c|}{ Proceso } & hi (\%) \\
\hline C) Control de calidad & $42,6 \%$ \\
\hline A) Diagnóstico & $24,1 \%$ \\
\hline E) Postservicio & $20,4 \%$ \\
\hline B) Presupuesto & $5,6 \%$ \\
\hline D) Estructura costos & $3,7 \%$ \\
\hline F) Otros & $3,7 \%$ \\
\hline TOTAL & $100 \%$ \\
\hline
\end{tabular}

Fuente: Elaboración propia.

Tabla N 11: Paso de los indicadores de gestión

\begin{tabular}{|c|c|c|}
\hline Proceso & Parámetro & $\begin{array}{c}\text { Valor o peso } \\
\text { del parámetro }\end{array}$ \\
\hline Diagnóstico & $a$ & 24,1 \\
\hline Control de calidad & $\beta$ & 42,6 \\
\hline Postservicio & $\Omega$ & 20,4 \\
\hline
\end{tabular}

Fuente: Elaboración propia

\section{RESULTADOS FINALES}

Modelo de indicadores de gestión basados en los procesos críticos para pequeñas empresas de mantenimiento de equipos pesados.

Con fundamento científico, se puede afirmar que las pequeñas empresas del rubro de mantenimiento de maquinaria y equipos pesados incrementarán su competitividad siempre y cuando dediquen recursos a mejorar la gestión en sus procesos críticos, es decir el diagnóstico, control de calidad y postservicio.

A estos tres procesos en la cadena de valor, se denominan críticos por ser considerados fuente generadora de competitividad. Claro está que el nivel de competitividad alcanzado está en medida de la profundidad con que se abarque cada proceso, tal como lo muestra la Tabla 12.

En base a la matriz de la Tabla 12 se califica el nivel de gestión de cada proceso y se asignan valores: $\mathrm{A}$, $B$ y $C$ para obtener el valor de $\mathrm{X}$.

$$
X=24,1(A)+42,06(B)+20,4(C)
$$


Tabla 12. Matriz de calificación de indicadores

A

B

C

\begin{tabular}{|c|c|c|c|}
\hline CALIFICAC. & DIAGNÓSTICO & CONTROL DE CALIDAD & POSTSERVICIO \\
\hline 1 & Superficial & Sin control & Sin comunicación, ni seguimiento \\
\hline 3 & Intermedio & Supervisor de calidad & Comunicación y seguimiento medio \\
\hline 6 & Profundo & Prueba de calidad & Comunicación y seguimiento constante \\
\hline
\end{tabular}

Fuente: Elaboración propia.

\section{CONCLUSIONES Y RECOMENDACIONES}

\subsection{Conclusiones}

- En Lima Metropolitana existen más de 11 mil empresas pequeñas, de las cuales aproximadamente 320 de ellas se dedican a brindar el servicio de mantenimiento de maquinaria y equipos pesados, caracterizadas por cumplir las necesidades de sus clientes, limitándose al plano técnico, no pudiendo ingresar al mercado de mayor poder adquisitivo oligopolizado por empresas de tamaños mayores.

- Los procesos principales en la cadena de valor de las empresas dedicadas al mantenimiento de maquinaria pesada son: 1) compras, 2) operaciones (diagnóstico, presupuesto, mantenimiento, control de calidad), 3) marketing y 4) postservicio; ya que son indispensables para obtener un resultado de calidad. Sin embargo, de todos ellos el diagnóstico, control de calidad y el postservicio son responsables del $80 \%$ de la competitividad que puede alcanzar una empresa de este rubro.

- Cada proceso crítico generará competitividad en la medida de la profundidad con que se gestionen; así se tienen tres niveles para cada proceso, el diagnóstico superficial, intermedio y profundo; el control de calidad ausente, supervisión de calidad y prueba de calidad; y por último el postservicio sin seguimiento, de seguimiento esporádico y de seguimiento constante.

- El nivel de profundidad al gestionar los procesos críticos $(X)$, y la competitividad reflejada en los ingresos ( $Y$ ) que alcanzan las empresas en estudio guardan fuerte relación directa; es decir, un aumento en $\mathrm{X}$, provoca un incremento en Y. Expresada matemáticamente dicha relación describe una ecuación de tipo exponencial, al que se reconoce como "Modelo basado en indicadores de gestión".

Tabla No 13: Modelo basado en indicadores de gestión

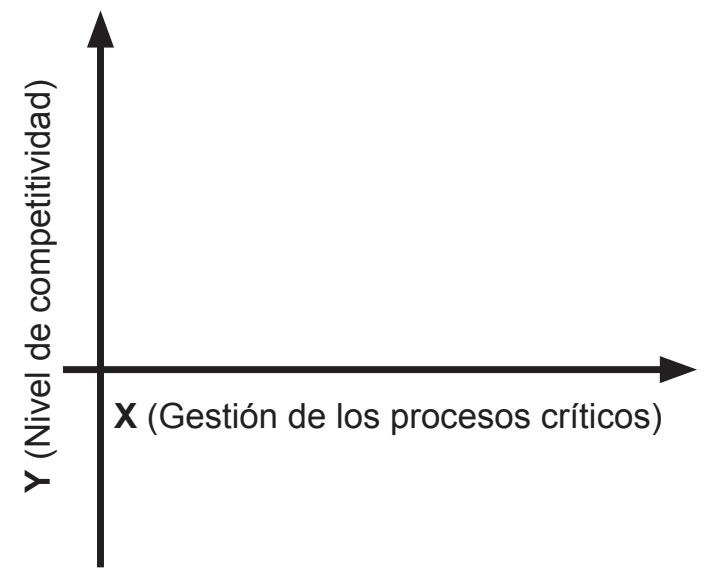

Fuente: Elaboración propia.

\subsection{Recomendaciones}

- Las empresas micro y pequeñas carecen de sofisticación en sus procesos; para no permanecer rezagadas por empresas de mayor tamaño deben invertir recursos en aplicar herramientas de gestión, esto generará competitividad independientemente de la actividad económica a la que se dediquen. Para dicho propósito el Estado debe participar concientizando, motivando y brindando a las mypes los conocimientos necesarios para el proceso de sofisticación del sector empresarial peruano. 
- Se recomienda que el modelo presentado basado en indicadores de gestión no solo debe ser empleado para medir el nivel de competitividad de las empresas, es decir la etapa del diagnóstico del problema; sino también debe ser aplicado como guía para la elaboración de planes y programas que busquen mejorar la calidad.

\section{REFERENCIAS BIBLIOGRÁFICAS}

[1] Adriani, C., Biasca, R y Rodríguez, M. (2003). El Nuevo Sistema de Gestión para las PYMES, a nivel de las empresas latinoamericanas: Grupo Editorial Norma. Madrid

[2] Durand Chahud, C. (2011). El Desarrollo de las MYPES y su impacto en la inclusión nacional. Revista Empresas y Negocios. $N^{\circ} 56$, Mayo. Lima.

[3] Escorza, P. y Vallas, J. (2003). Tecnología e innovación en la empresa. Barcelona: Ediciones UPC.

[4] Federación Nacional de Pequeñas y Medianas Empresas Industriales del Perú. FENAPI
(2005). Análisis de Competitividad de las Empresas. Lima.

[5] Ferreyros: www.ferreyros.com.pe

[6] Instituto Nacional de Estadística e Informática (2008). IV Censo Económico Nacional, Primeros Resultados.

[7] INEI: www.inei.gob.pe

[8] Jacobs Gálvez, L. (2009). Avance y Retos en la Promoción y Desarrollo de las MYPES. Revista de Trabajo. Julio, Lima.

[9] Newbold, P., Carlson, W. y Thorne B. (2008). Estadística para Administración y Economía. 6. ${ }^{a}$ ed. Pearson Educación: Madrid.

[10] Peñaranda: www.volvopenaranda.com.pe

[11] Porter, M (2010). Competitiveness: A New Economic Strategy for Peru. Conferencia magistral en la CADE 2010 en la Ciudad del Cusco-Lima-Perú.

[12] SUNAT: www.sunat.gob.pe 